\title{
9
}

\section{Optimal Design and Steady-State Operation} Benoît Chachuat ${ }^{1}$

\section{1}

\section{Introduction}

The focus of this chapter is on describing procedures for the optimal design and operation of microfabricated power generation processes. In contrast to the problem of selection of alternatives, as described earlier in Chapter [Selection of Alternatives and Process Design], a fixed process structure is considered throughout this chapter.

In many applications, the power demand remains essentially constant during the operation, with rapid changeovers, and, therefore, steady-state operation must be considered thoroughly. For a given power demand, or a power demand varying in a specified range, the design and steady-state operation problem is to determine values of the design variables (e.g., sizes of the individual components such as fuel processing reactor and fuel cell) as well as operational variables (e.g., fuel flow rates and operating temperature) so as to maximize its (steady-state) performance, in light of safety, reliability, as well as other considerations.

In other applications, the power demand may change rapidly and the devices may be operated periodically, with frequent start-ups and shut-downs. In this case, special attention must be paid to the dynamics of these processes, in addition to their steady-state operation. Ideally, one would like to optimize the design and operation over an entire operation cycle, including start-up, steady-state operation, and shut-down. However, because the duration and power demand profile of a mission is rarely known in advance, such an optimization is hardly tractable. Instead, the start-up phase can be optimized separately from the subsequent steady-state operation. For start-up purpose, the devices will most likely be coupled with a small battery or ultra-capacitor, whose role will be to ensure that the power demand is met when the fuel cell is unavailable or can only satisfy part of the demand, and to provide the

1) Corresponding author. Laboratoire d'Automatique, École Polytechnique Fédérale de Lausanne, Station 9, CH-1015 Lausanne, Switzerland. Tel: +41 21693 3844, Fax: +41216932574 
energy needed to heat the fuel cell stack up to a temperature at which chemical and electrochemical reactions are fast enough. That is, the objective of the optimal start-up problem is to bring the fuel cell to a desired operating point (steady state), in a manner that minimizes the mass of fuel and battery required, while meeting the nominal power demand at all times, along with operational restrictions and safety requirements.

At the micro scale, a different paradigm to the unit-operations paradigm of macro-scale process design and operation is necessary. The reason for this is that the different units constituting a microfabricated system are tightly spatially integrated and can no longer be considered to operate independently from each other. Accordingly, operational decisions must be taken at an early stage of development, together with design decisions. For example, increasing the operating temperature of a microfabricated reactor increases the heat losses per unit surface area, but because the reaction rates are also enhanced, the volume needed to achieve a given conversion is reduced. In the case where the latter effect dominates, one then obtains the counter-intuitive result that increasing the operating temperature lowers the overall heat losses for the system. In other words, it is of paramount importance to determine the operation policy simultaneously with the design and sizing of the units.

Because the underlying physicochemical phenomena are complicated and intrinsically coupled, one cannot rely only on engineering intuition to find out the optimal design and operation. The use of mathematical models along with systematic optimization methods based on mathematical programming is clearly warranted. Because optimization algorithms may require hundreds, or even thousands, of iterations to converge, fast, reliable and robust solution of the models is needed. These considerations proscribe the use of computationally expensive models based on Computational Fluid Dynamics (CFD), as described earlier in Chapter [Reaction Engineering and Detailed Modeling]. System-level models, as presented in Chapter [Selection of Alternatives and Process Design], are not adequate either, because they cannot represent the couplings between design and operational decisions.

In this chapter, the focus is on the so-called intermediate-fidelity models. These models are spatially distributed, rely on validated kinetic expressions, and allow optimization of unit sizes and operation for a given process structure without the need to specify a detailed geometry. This level of modeling detail is especially useful for technologies with a demonstrated proof of principle.

In the remainder of this chapter, first the formulation of intermediatefidelity models is discussed (Section 9.2). Then, the problem of optimal design and operation is addressed, both in the case of a nominal power demand (Section 9.3) and of a variable power demand (Section 9.4). The emphasis is placed on explaining the problem formulations and illustrating the benefit of these 
approaches through a number of case studies; the concrete techniques used to solve these problems are not explained.

\section{2}

\section{Models for Optimal Design and Operation}

The devices that are considered for man-portable power generation involve complex geometries, multiple scales, time dependence, and parametric uncertainty. Therefore, with current computational capabilities and available algorithms, the optimal design and operation problem cannot be solved in a single step. The system-level approach described earlier in Chapter [Selection of Alternatives and Process Design] (see also [19,20]), allows to compare between many different technology alternatives for man-portable power generation based on a process superstructure, including thousands of different designs. Although it allows identifying conditions under which the technologies considered are a promising alternative to batteries, such an approach has currently the limitation that it requires values to be set for some modeling parameters that in principle can be calculated, e.g., fuel cell efficiency, conversions, etc. Furthermore, it cannot predict optimal values for several key operating parameters, such as the operating temperature and the fuel/air ratios, nor does it consider emissions of trace components, such as carbon monoxide, ammonia, or nitric oxide.

At the other extreme, the development of CFD models is a very versatile tool for detailed analysis (see Chapter [Reaction Engineering and Detailed Modeling]; compare [11,14]) as well as justification of modeling assumptions [17]. However, CFD requires comprehensive knowledge of the detailed geometric design, and an extensive modeling effort. Moreover, it can be very computationally expensive. Consequently, the applicability of CFD to optimization of the design and operation is currently rather limited.

The foregoing considerations motivate the development of models of an intermediate detail level to study the optimal sizing of units and optimal operation. In order for the couplings between the design and operational decisions to be accurately represented, these models rely on first principles and include detailed kinetic mechanisms. Spatial dependence is considered whenever necessary, but, unlike CFD, a fully defined geometry is not required. Rather, a minimal number of design parameters needs to be specified, such as the volume and the surface-area-to-volume-ratio of the units. Intermediate fidelity models have been initially developed in the context of steady-state operation [8,27], which results in models that are comprised of mixed sets of differential and algebraic equations (DAEs). In the case of transient operation, integration through time must be considered in addition to the spatial dis- 
tribution, which leads to models that consist of partial differential-algebraic equations (PDAEs) [4,9]. Only steady-state aspects shall be discussed in this chapter. The design, operation, and control issues associated with the transient operation of man-portable power generation processes are addressed in subsequent Chapters [Design of Hybrid Electrochemical Devices] and [Control].

Obviously, the nature of intermediate-fidelity models is dependent on the class of devices considered. Valid approximations can be established by detailed modeling, scaling analysis, and experimental evidence. Typical simplifications, which are valid for a specific class of devices only, are given for illustrative purpose next. For high-temperature systems with maximal characteristic dimensions in the order of millimeters and high thermal conductivity, a good approximation is to assume a spatially uniform temperature. In practice, the use of silicon, together with the presence of a catalyst support, ensures such a high thermal conductivity [17]. Yet the approximation of uniform temperature may not be adequate for all microstructured reactors, e.g., in the case of combustion [21]. Besides temperature, a one-dimensional distribution of the species balance appears to be an adequate approximation for reactors that are made of thin tubes, e.g., for tubes with diameters in the order of $100 \mu \mathrm{m}$ [16].

The formulation of an intermediate-fidelity model for a simple man-portable power generation process is presented in the following case study.

\section{Case Study}

A micro power generation process that consists of a fuel processing reaction, a solid-oxide fuel cell (SOFC) and two burners, microfabricated into a single silicon stack fed with ammonia and butane fuels is studied. This technology alternative satisfies three important criteria, namely: (i) the potential for high performance, which is ascertained from system-level considerations; (ii) a demonstrated proof of concept; and (iii) the availability of validated chemical kinetics. Here, the SOFC is chosen because of the long-term promise for fuel flexibility [23]. Although butane partial oxidation combined with a SOFC is expected to have higher energy density, the corresponding chemistry has not yet been sufficiently demonstrated in microreactors. On the other hand, ammonia decomposition has been successfully performed with conversions exceeding $90 \%[2,11]$. This motivated the choice of ammonia as primary fuel, despite the limitation of potential applications due to its toxicity. Moreover, butane is chosen as secondary fuel because of its high energy density, which makes it a suitable heat source.

The process is arranged in two lines (Fig. 9.1). The ammonia line begins with a reactor for the catalytic decomposition of ammonia $\left(\mathrm{NH}_{3}\right)$ to produce hydro- 


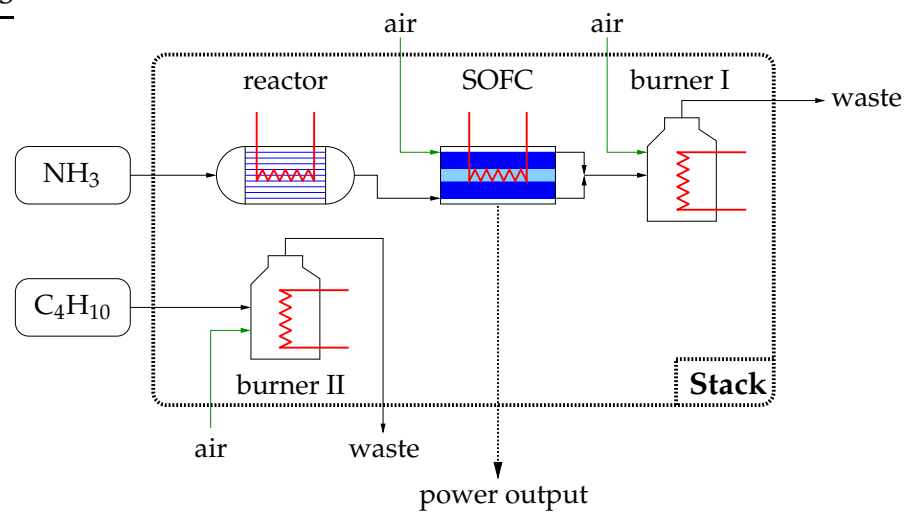

Fig. 9.1: Conceptual process flow-sheet.

gen $\left(\mathrm{H}_{2}\right) . \mathrm{H}_{2}$ is fed to the SOFC anode while air is fed to the SOFC cathode, and electrical power is produced from the electrochemical reaction. The effluents from the anode and cathode compartments of the SOFC are then passed to Burner I for catalytic oxidation of residual $\mathrm{H}_{2}$ and $\mathrm{NH}_{3}$. The butane line consists of Burner II, which is fed with a mixture of butane $\left(\mathrm{C}_{4} \mathrm{H}_{10}\right)$ and air in order to produce heat from catalytic oxidation.

A summary of the main assumptions used to formulate an intermediatefidelity model for this process is given hereafter. For a complete statement of the model equations, please refer to $[8,27]$.

Intermediate-Fidelity Model The main assumptions used in the model formulation are the following:

A1. The pressure inside the stack is uniform and equals the atmospheric pressure

This assumption requires that the pressure drop along the gas channels remain relatively small, which is typically the case for characteristics length as small as $50 \mu \mathrm{m}$ in the radial direction and a few $\mathrm{cm}$ in the axial direction.

A2. The gas phase is ideal

This assumption appears to be reasonable when the stack operates at elevated temperature and atmospheric pressure.

A3. The stack operates at a spatially uniform temperature

This assumption requires that heat transfer is fast enough, which is typically the case at the micro scale for silicon-based reactors due to the high thermal conductivity of silicon and the small length scales involved. 
A4. The molar fluxes in the gas channels of the four units are convective in the flow direction (no axial diffusion) and radial gradients are neglected This last assumption asserts that microfabricated units such as reactors or fuel cells can be approximated well by an idealized model using 1-D distributed models. Neglecting axial diffusion is motivated by the fact that the Peclet number in the axial direction is relatively large; neglecting radial gradients, on the other hand, appears to be a legitimate assumption since the characteristic time for diffusion is generally smaller than the characteristic time for reaction.

Based on Assumptions A1-A4, the mass and species equations for each unit can be written as that of a plug-flow reactor (PFR) at steady-state [13]:

$$
\begin{aligned}
& \frac{d F}{d \eta}=V \sum_{j=1}^{n_{r}} \sum_{i \in \mathcal{I}_{s}} v_{i, j} r_{j} \\
& \frac{d z_{i}}{d \eta}=\frac{V}{F} \sum_{j=1}^{n_{r}}\left[v_{i, j}-z_{i} \sum_{k \in \mathcal{I}_{s}} v_{k, j}\right] r_{j}, \quad i \in \mathcal{I}_{s} .
\end{aligned}
$$

In these equations, $\eta \in[0,1]$ stands for the dimensionless coordinate in the flow direction; $V$ is the volume of the unit; $T$ is the operating temperature; $F$ is the total molar flow; $z_{i}$ is the mole fraction of species $i ; r_{j}$ is the reaction $j ; v_{i, j}$ is the stoichiometric coefficient for species $i$ in reaction $j$; $\mathcal{I}_{s}:=\left\{\mathrm{NH}_{3}, \mathrm{C}_{4} \mathrm{H}_{10}, \mathrm{H}_{2}, \mathrm{H}_{2} \mathrm{O}, \mathrm{O}_{2}, \mathrm{~N}_{2}, \mathrm{NO}, \mathrm{CO}_{2}\right\}$; and $n_{r}$ is the number of reactions. In addition to the differential equations $(9.1,9.2)$, the unit models contain algebraic equations to calculate the reaction rates $r_{j}$ as a function of the molar fractions $z_{i}, i \in \mathcal{I}_{s}$, as well as operational variables such as the operating temperature $T$ or the fuel cell voltage $U$; some of these algebraic equations are explicit (e.g., the reaction rates), and some are implicit (e.g., the Butler-Volmer equations). Overall, each unit is thus described by a set of DAEs.

By putting the DAEs describing each unit together, one obtains a global model for the fuel cell stack in the form of multi-stage DAEs, wherein each stage corresponds to a given unit. The solutions of the equations for each stage in the ammonia line are coupled: the mole fractions at the exit of the reactor affect the initial conditions for the DAEs describing the SOFC; in turn, the mole fractions at the exit of the SOFC affect the initial conditions for the DAEs describing Burner I.

The steady-state operation and decision variables in each unit of the fuel cell stack can be summarized as follows:

Ammonia Line:

1. Reactor: $\mathrm{NH}_{3}$ is endothermically decomposed using a catalyst according to the overall reaction:

$$
\mathrm{NH}_{3} \rightarrow \frac{3}{2} \mathrm{H}_{2}+\frac{1}{2} \mathrm{~N}_{2} \text {. }
$$


The reduced kinetic rate expressions proposed by [10] for ammonia decomposition over ruthenium (temperature dependent) can be used as a first approximation. In the reactor model, the reactor volume, $V^{\mathrm{r}}$, is a design decision variable; the optimal $\mathrm{NH}_{3}$ inlet flow rate, $F_{\mathrm{in}}^{\mathrm{r}}$, together with the operating temperature are operational decision variables.

2. SOFC: The solid-oxide fuel cell consists of a cathode and an anode that are separated by a solid electrolyte. In the cathode compartment, oxygen from the air is converted to oxygen ions. The oxygen ions migrate to the anode side through the ion-conducting electrolyte. In the anode compartment, hydrogen reacts with oxygen ions to produce water. Electrons flow back to the cathode via an external circuit. The half cell reactions of the process are:

$$
\text { cathode: } \frac{1}{2} \mathrm{O}_{2}+2 \mathrm{e}^{-} \rightarrow \mathrm{O}^{2-}, \quad \text { anode: } \mathrm{H}_{2}+\mathrm{O}^{2-} \rightarrow \mathrm{H}_{2} \mathrm{O}+2 \mathrm{e}^{-} \text {. }
$$

The open-circuit potential for the SOFC is taken as the Nernst potential. Given the operating voltage of the fuel cell, $U$, one can then calculate the electrical current density, $j$. This can be done by accounting for the irreversibilities arising from both ohmic losses through the electrolyte [5] and activation polarizations at the anode and cathode sides [1]; on the other hand, it was shown that irreversibilities in the form of concentration overpotentials at both the anode and cathode sides can be neglected [8]. The rate of electrochemical reaction is obtained from the electrical current density per Faraday's law. Further, the electrical power generated by the fuel cell, $\mathcal{P}$, can be calculated as:

$$
\mathcal{P}:=\frac{A^{\mathrm{fc}} V^{\mathrm{fc}}}{2} U \int_{0}^{1} j d \eta,
$$

with $V^{\mathrm{fc}}$ standing for the fuel cell volume, and $A^{\text {fc }}$ being the surfacearea-to-volume ratio of the anode and cathode compartments for the electrochemical reaction. In the SOFC model, the fuel cell volume is a design decision variable; the air inlet flow rate, $F_{\mathrm{in}}^{\mathrm{ca}}$, the fuel cell voltage and the operating temperature are operational decision variables.

3. Burner I: The effluents from the SOFC anode and cathode are passed to burner I, possibly with an additional air stream. Catalytic oxidation of the residual $\mathrm{H}_{2}$ and $\mathrm{NH}_{3}$ can provide heat to balance heat losses as well as the endothermic decomposition of $\mathrm{NH}_{3}$ in the reactor; part of the residual $\mathrm{NH}_{3}$ is also decomposed into $\mathrm{H}_{2}$. The overall reactions oc- 
8 8 9 Optimal Design and Steady-State Operation

curring in Burner I are:

$$
\begin{aligned}
\mathrm{NH}_{3}+\frac{5}{2} \mathrm{O}_{2} & \rightarrow \mathrm{NO}+\frac{3}{2} \mathrm{H}_{2} \mathrm{O}, & \mathrm{NH}_{3}+\frac{3}{2} \mathrm{NO} & \rightarrow \frac{5}{4} \mathrm{~N}_{2}+\frac{3}{2} \mathrm{H}_{2} \mathrm{O}, \\
\mathrm{NH}_{3} & \rightarrow \frac{1}{2} \mathrm{~N}_{2}+\frac{3}{2} \mathrm{H}_{2}, & \mathrm{H}_{2}+\frac{1}{2} \mathrm{O}_{2} & \rightarrow \mathrm{H}_{2} \mathrm{O} .
\end{aligned}
$$

The kinetic rate expressions proposed by [22] (temperature dependent) can be used to model these reactions as a first approximation. At the temperatures of operation of the fuel cell (around 1000-1300K), the unimolecular decomposition of NO is not experimentally observed, therefore it is ignored in the model. In the burner I model, the burner volume, $V^{\mathrm{b}_{\mathrm{I}}}$, is a design decision variable; the burner inlet air flow rate, $F_{\text {in }}^{\mathrm{b}_{\mathrm{I}}}$, and the operating temperature are operational decision variables.

Butane Line:

4. Burner II: The combustion of $\mathrm{C}_{4} \mathrm{H}_{10}$ supplies additional heat to balance the heat losses to the ambient and the endothermic decomposition of $\mathrm{NH}_{3}$. A premixed butane/air mixture is fed to the burner, with an oxygen stoichiometry value of 1.2 so that the combustion occurs with excess air. Butane combustion is modeled as a homogeneous irreversible, onestep reaction:

$$
\mathrm{C}_{4} \mathrm{H}_{10}+\frac{13}{2} \mathrm{O}_{2} \rightarrow 4 \mathrm{CO}_{2}+5 \mathrm{H}_{2} \mathrm{O}
$$

As a first approximation, the kinetic rate expression proposed by [26] can be used. In the burner II model, the volume of the burner, $V^{\mathrm{b}_{\mathrm{II}}}$, is a design decision variable; the inlet flow rate of butane, $F_{\text {in }}^{\mathrm{b}_{\text {II }}}$, and the operating temperature are operational decision variables.

For simulation of the intermediate-fidelity model, in addition to all chemical and thermodynamical properties, inlet compositions and parameters (for which the values as in [8] are used herein), one needs to specify ten degrees of freedom. Four of them correspond to design decisions: the volumes $V^{\mathrm{r}}$, $V^{\mathrm{fc}}, V^{\mathrm{b}_{\mathrm{I}}}$ and $V^{\mathrm{b}_{\mathrm{II}}}$ of the reactor, fuel cell, burner I and burner II, respectively; the remaining six degrees of freedom correspond to operational decisions: the operating temperature $T$, the fuel cell voltage $U$, the ammonia inlet flow rate $F_{\text {in }}^{\mathrm{r}}$ to the reactor, the air inlet flow rates $F_{\text {in }}^{\mathrm{ca}}$ to the fuel cell cathode and $F_{\text {in }}^{\mathrm{b}_{\mathrm{I}}}$ to burner I, and the butane inlet flow rate $F_{\text {in }}^{\mathrm{b}_{\text {II }}}$ to burner II.

Steady-State Simulations Simulation results are presented to illustrate the operation of the ammonia line. The following unit volumes are used: $V^{\mathrm{r}}=$ $V^{\mathrm{b}_{\mathrm{I}}}=9.6 \times 10^{-10} \mathrm{~m}^{3}$ and $V^{\mathrm{fc}}=9.6 \times 10^{-8} \mathrm{~m}^{3}$. Observe that the fuel cell 
volume (i.e., the sum of both anode and cathode compartments) is taken to be a hundred times larger than either the reactor and burner I volumes since the electrochemical reactions are much slower than the ammonia decomposition and hydrogen/ammonia oxidation reactions. Furthermore, the inlet flow rates of ammonia to the reactor and air to the cathode and burner I are set to: $F_{\text {in }}^{\mathrm{r}}=15 \mathrm{sccm}, F_{\text {in }}^{\mathrm{ca}}=100 \mathrm{sccm}$, and $F_{\text {in }}^{\mathrm{b}_{\mathrm{I}}}=0 \mathrm{sccm}$. This latter value indicates that no additional air is fed into burner I, assuming that the fuel cell is operated at a high oxygen excess and thus enough residual oxygen remains in burner I's feed. Finally, an operating temperature $T=1300 \mathrm{~K}$ and a cell voltage $U=0.65 \mathrm{~V}$ are considered. These specifications were chosen since they correspond to a production of electrical power close to $\mathcal{P}=1 \mathrm{~W}$.

The component mole fractions along the gas channels in the reactor, anode, cathode and burner I are presented in Fig. 9.2. Based on the gas molar flow rates (not shown on Fig. 9.2) and compositions, performance factors can be easily calculated for each unit of the device. It should be mentioned that these factors can be used as parameters at the system level (see Chapter [Selection of Alternatives and Process Design]), and the intermediate-fidelity model can thus be used as a tool to estimate their values or for validation purposes.

- The conversion of ammonia in the reactor is calculated to be $\zeta_{\mathrm{NH}_{3}}^{\mathrm{r}} \approx$ 0.987. This value indicates that most of the ammonia is being converted into hydrogen.

- The micro SOFC operation can be monitored by considering three performance factors, namely the hydrogen conversion in the anode compartment, $\zeta^{\text {an }}$, the air excess number in the cathode compartment, $\Phi^{\mathrm{ca}}$, and the fuel cell efficiency, $\eta^{\mathrm{fc}}$. The latter factor accounts for the losses induced by the irreversibilities in the fuel cell (i.e., $\eta^{\mathrm{fc}}=100 \%$ when no current is passed through the fuel cell). The following values are obtained for the conditions shown in Fig. 9.2: $\zeta^{\text {an }} \approx 0.503, \Phi^{\mathrm{ca}} \approx 3.76$, and $\eta^{\mathrm{fc}} \approx 0.688$. These values indicate that neither the available hydrogen nor the supplied oxygen are limiting for the electrochemical reaction, but the hydrogen conversion is low, mainly due to an insufficient residence time in the anode compartment. Furthermore, the high SOFC efficiency indicates that the irreversibilities remain limited.

- The conversions of hydrogen and ammonia in burner I are calculated to be $\zeta_{\mathrm{H}_{2}}^{\mathrm{b}_{\mathrm{I}}} \approx 0.516$ and $\zeta_{\mathrm{NH}_{3}}^{\mathrm{b}_{\mathrm{I}}} \approx 0.999$. The latter value shows that the conversion of ammonia is nearly complete, whereas only approximately half of the residual hydrogen is converted from the former.

This rapid analysis reveals that the process does not operate very efficiently under these specific conditions, as a large fraction of the hydrogen produced in the reactor is discarded from the device without being used. Moreover, 


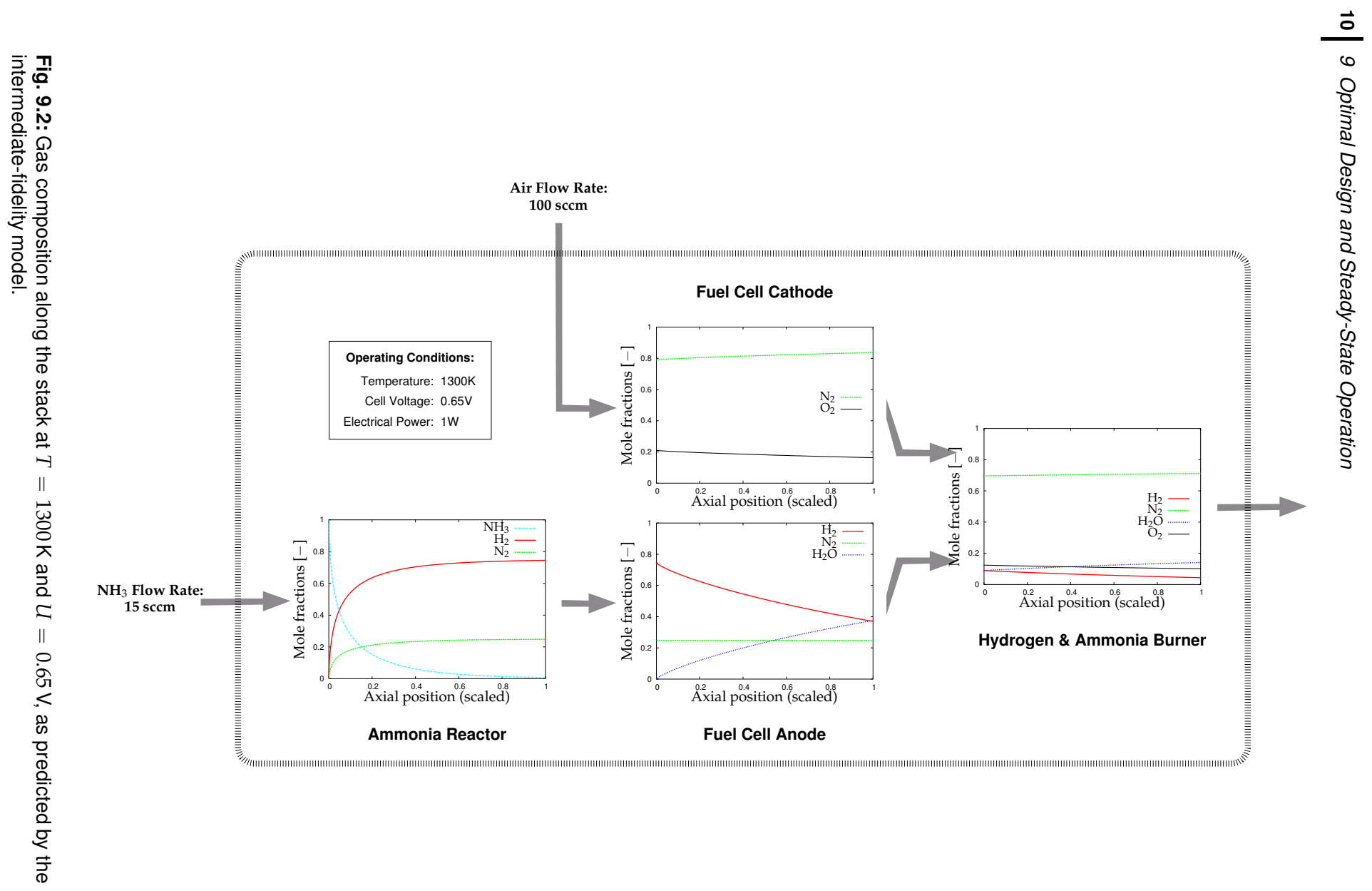


the residual mole fraction of nitric oxide is relatively high, in the order of $1500 \mathrm{ppm}$, hence greatly exceeding the currently allowed safe level (neither the concentration of $\mathrm{NH}_{3}$ nor that of $\mathrm{NO}$ are represented in Fig. 9.2 because their values are much lower than those obtained for the other components $\mathrm{H}_{2}$, $\mathrm{H}_{2} \mathrm{O}, \mathrm{N}_{2}$ and $\mathrm{O}_{2}$ ). Accordingly, the process performance could be greatly improved under the specification of more suitable design and operational variable values. These aspects are the focus of the following two sections.

\section{3}

\section{Optimal Design and Steady-State Operation for Nominal Power Demand}

Intermediate-fidelity models are invaluable tools in the design of man-portable power generation systems since they provide a picture of the gas composition and velocities, potential and current density in the system for various process configurations and operating conditions. Not only can such models be used to examine the sensitivity of the system with respect to relevant design parameters and operational variables, but this sensitivity information can also be used to improve the system performance through the application of systematic optimization methods.

Qualitatively, the optimization problem for optimal design and steady-state operation of man-portable power generation processes can be stated as follows:

"Determine the design and operational decisions that maximize the device performance, while meeting the nominal power demand, guaranteeing autothermal operation, and respecting the safety and operational constraints."

Because intermediate fidelity models are comprised of multistage DAEs, this formulation gives rise to challenging, constrained dynamic optimization problems. Efficient methods exist for the numerical solution of such problems [6], yet their description lies beyond the scope of this chapter.

A number of remarks are in order:

- The decision variables in the optimization problem correspond to the design and operational variables in the intermediate-fidelity model (see Section 9.2). In particular, optimal values for both sets of variables are determined simultaneously.

- Important metrics for the comparison of different design and operational alternatives are those expressed in terms of the energy density of the system (see Chapter [Selection of Alternatives and Process Design]; compare [18]). Typically, the fuel energy density - defined as the electrical energy produced per unit mass of fuel, is considered as the performance metric for optimal design and operation. Note that neither the weights 
of the power generation device nor fuel containers are taken into account in this metric, which is therefore best suited for systems where the mass of the fuel dominates (e.g., for long mission durations). Of course, the proposed design and operation methodology is flexible enough that it allows optimization of other metrics [18], such as the overall system energy density for a given mission duration, or the energy efficiency defined as the power output divided by the product of heating values and molar flow rates.

- Implicit to the optimization formulation is the ability of the intermediatefidelity model to predict (i) the electrical power produced by the fuel cell, and (ii) the total heat load on the device, for given values of the design and operational variables. Besides meeting the nominal power demand and operating autothermally, the device is typically required to satisfy a number of safety and/or operational constraints. Safety constraints can be defined to limit the emissions of trace components, such as ammonia or nitric oxide, that have potential impact either on human health or on the environment. For example, these limits can be defined by following the U.S. Occupational Safety and Health Administration (OSHA) regulations $^{2}$ or the American Conference of Governmental Industrial Hygienists (ACGIH) recommendations ${ }^{3}$. For structural stability reasons (see Chapter [Structural Considerations] and [25]), the maximum allowable operating temperature may be constrained. Operational constraints may also include limits on the gaseous flow rates in the micro channels, as well as restrictions on the cell potential depending on the application.

Once an optimal design and operation strategy has been determined that maximizes the device performance and satisfies the constraints, a rather natural question that arises is how much confidence can be placed into that strategy? Many sources of uncertainty can indeed invalidate an optimal strategy. Typically, the mathematical expressions used to calculate the reaction rates in an intermediate-fidelity model carry a lot of uncertainty. Moreover, because the geometry of the units is usually not known precisely, certain model parameters may be highly uncertain, such as the catalyst support surface-areato-volume ratios or the overall heat transfer and emissivity coefficients. Postoptimal sensitivity analysis is a systematic way of analyzing the influence of uncertainty on an optimal strategy $[7,12]$. The idea therein is to calculate the variation in the system performance and the optimal design and operation variables incurred by (infinitesimally small) variations in the uncertain parameters. Also, when large parameter variations are considered, parametric

2) http://www.osha.gov/SLTC/.

3) http://www.acgih.org/TLV/. 
programming techniques can be used to monitor the change in optimal solution over the entire parameter range [3].

Besides analyzing the effect of uncertain parameters, parametric studies also prove useful for studying the influence of various design choices or operating conditions [8]. For example, by varying the nominal power demand and monitoring the corresponding change in performance, one can identify the applications for which the considered technology will most likely be best suited. Regarding design choices, parametric studies can help answer outstanding questions such as: Which reaction kinetics are to be improved in priority for enhancing the system performance? Does the electrolyte thickness have a large influence on the system performance? Is heat recovery between inlet and outlet streams a worth considering option?

The aforementioned considerations are illustrated by a case study subsequently, which is the continuation of the case study presented in Section 9.2.

\section{Case Study (Continued)}

Consider the micro power generation process shown in Fig. 9.1, for which an intermediate-fidelity model has already been described. The optimal design and operation problem is to determine values of the design variables $V^{\mathrm{r}}, V^{\mathrm{fc}}$, $V^{\mathrm{b}_{\mathrm{I}}}$ and $V^{\mathrm{b}_{\text {II }}}$ and operational variables $T, U, F_{\mathrm{in}}^{\mathrm{r}}, F_{\mathrm{in}}^{\mathrm{ca}}, F_{\mathrm{in}}^{\mathrm{b}_{\mathrm{I}}}, F_{\text {in }}^{\mathrm{b}_{\text {II }}}$ that

maximize : fuel energy density

subject to : nominal power demand

autothermaloperation maximum $\mathrm{NH}_{3}$ and $\mathrm{NO}$ emissions.

The objective and constraint functions in this optimization problem are as follows:

- The fuel energy density, $e_{\text {dens' }}^{\text {fuel }}$ is calculated as:

$$
e_{\text {dens }}^{\text {fuel }}=\frac{\mathcal{P}^{\text {nom }}}{M W_{\mathrm{NH}_{3}} F_{\text {in }}^{\mathrm{r}}+M W_{\mathrm{C}_{4} \mathrm{H}_{10} F_{\text {in }}^{\mathrm{b}_{\text {iI }}}}}
$$

where $\mathcal{P}^{\text {nom }}$ denotes the nominal power demand; $M W_{\mathrm{NH}_{3}}$ and $M W_{\mathrm{C}_{4} \mathrm{H}_{10}}$ are the molecular weights of ammonia and butane, respectively. Observe, in particular, that maximizing the fuel energy density is equivalent to minimizing the inlet mass flow of ammonia and butane in the system (approx. $F_{\mathrm{in}}^{\mathrm{r}}+3.4 F_{\text {in }}^{\mathrm{b}_{\mathrm{II}}}$ ).

- The nominal demand constraint simply reads:

$$
\mathcal{P}-\mathcal{P}^{\text {nom }}=0,
$$

with the electrical power $\mathcal{P}$ produced by the SOFC calculated per (9.3). 
- The autothermal operation constraint is given by:

$$
\left(\dot{H}_{\text {in }}^{\mathrm{r}}+\dot{H}_{\text {in }}^{\mathrm{ca}}+\dot{H}_{\text {in }}^{\mathrm{b}_{\mathrm{I}}}-\dot{H}_{\text {out }}^{\mathrm{b}_{\mathrm{I}}}\right)+\left(\dot{H}_{\text {in }}^{\mathrm{b}_{\text {II }}}-\dot{H}_{\text {out }}^{\mathrm{b}_{\text {II }}}\right)-\dot{Q}^{\text {loss }}-\mathcal{P}=0 .
$$

Here, $\dot{H}_{\mathrm{in}}^{\mathrm{r}}, \dot{H}_{\mathrm{in}}^{\mathrm{ca}}, \dot{H}_{\mathrm{in}}^{\mathrm{b}_{\mathrm{I}}}, \dot{H}_{\text {out }}^{\mathrm{b}_{\mathrm{I}}}$ stand for the inlet and outlet enthalpy flows along the ammonia line, and $\dot{H}_{\text {in }}^{\mathrm{b}_{\text {II }}}, \dot{H}_{\text {out }}^{\mathrm{b}_{\text {II }}}$, for the inlet and outlet enthalpy flows along the butane line. They are calculated from the molar enthalpies for pure components and the gas composition and velocities determined by the intermediate-fidelity model. It is assumed that heat recovery between the inlet and outlet gas stream is possible. E.g. in the case of half heat recovery, the outlet stream temperature, $T^{\text {out }}$, is equal to $\frac{1}{2}\left(T-T^{\mathrm{amb}}\right)$, with $T^{\mathrm{amb}}=298 \mathrm{~K}$ the ambient temperature. Although difficult, note that heat recovery has already been demonstrated in micro chemical systems [2].

$\dot{Q}^{\text {loss }}$ stands for the overall heat losses to the environment, which accounts for conductive/convective as well as radiative heat losses. In the calculation of $\dot{Q}^{\text {loss }}$, a fixed aspect ratio, $A^{\mathrm{dev}}$, is assumed for the device, e.g., $A^{\text {dev }}=6\left(V^{\mathrm{r}}+V^{\mathrm{fc}}+V^{\mathrm{b}}+V^{\mathrm{bII}}\right)^{2 / 3}$ in the case of a cubic box.

- The emission constraints on $\mathrm{NH}_{3}$ and $\mathrm{NO}$ read:

$$
\begin{aligned}
y_{\mathrm{NH}_{3}, \text { out }}^{\mathrm{b}_{\mathrm{I}}} & \leq y_{\mathrm{NH}}^{\max } \\
y_{\mathrm{NO}, \text { out }}^{\mathrm{b}_{\mathrm{I}}} & \leq y_{\mathrm{NO}}^{\max },
\end{aligned}
$$

where the residual molar fractions $y_{\mathrm{NH}_{3}, \text { out }}^{\mathrm{b}_{\mathrm{I}}}$ and $y_{\mathrm{NO} \text {,out }}^{\mathrm{b}_{\mathrm{I}}}$ are calculated via the intermediate-fidelity model. The OSHA imposes threshold values of $y_{\mathrm{NH}_{3}}^{\max }=50 \mathrm{ppm}$ and $y_{\mathrm{NO}}^{\max }=25 \mathrm{ppm}$, respectively, for these species. However, the ACGIH recommends a tighter Time Weighted-Average (TWA) value of $y_{\mathrm{NH}_{3}}^{\max }=25 \mathrm{ppm}$ in regard to ammonia emissions, which is used instead. It should be noted that the aforementioned threshold limit values are very conservative since they correspond to exposure levels in a typical work environment.

Optimal design and operation results for this process are described below.

Optimal Design and Operation for a Nominal Power Demand $\quad$ For $\mathcal{P}^{\text {nom }}=1 \mathrm{~W}$ and in the absence of constraints on the operating temperature, it is found the fuel energy density is maximized for $T=1445 \mathrm{~K}$. Operating at such a high temperature is however unrealistic from a practical point of view, mainly because of material stability considerations [25]. Therefore, the operating temperature is removed from the list of decision variables, and a parametric study is carried out by varying its value in a more appropriate range, $T \in[1000 \mathrm{~K}, 1300 \mathrm{~K}]$. 

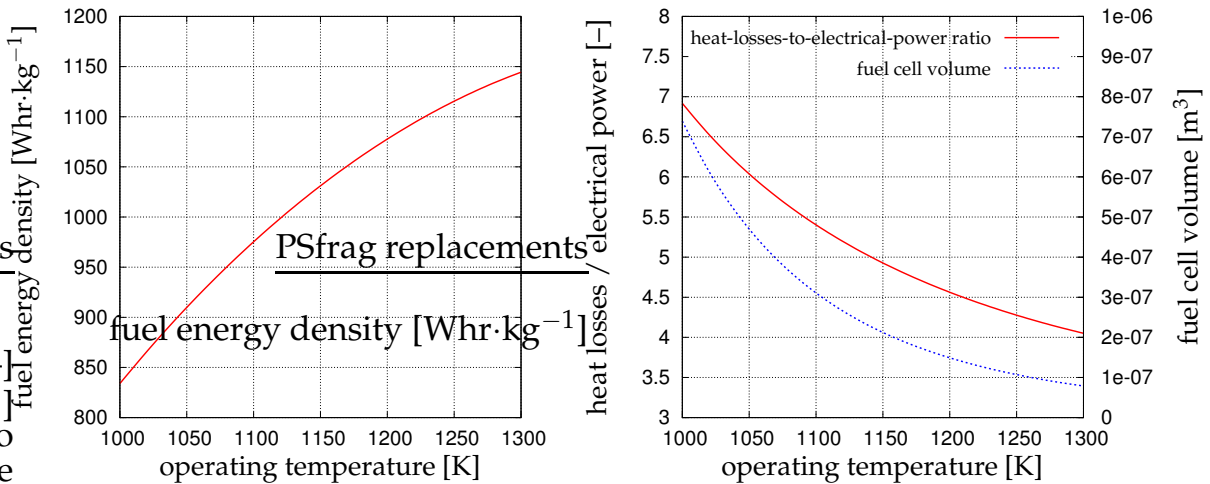

Fig. 9.3: Maximum fuel energy density (left plot), optimal heat-losses-to-electrical-power ratio (right plot), and optimal fuel cell volume (right plot) vs. operating temperature, for $\mathcal{P}^{\text {nom }}=1 \mathrm{~W}$.

The left plot of Fig. 9.3 shows the maximal fuel energy density that is achievable by the process, at steady-state, as a function of the operating temperature. Observe that the maximum achievable energy density is very sensitive to the operating temperature as it varies from $840 \mathrm{Whr} \cdot \mathrm{kg}^{-1}$ at $1000 \mathrm{~K}$, up to $1150 \mathrm{Whr} \cdot \mathrm{kg}^{-1}$ at $1300 \mathrm{~K}$. For the sake of comparison, recall that state-of-theart primary batteries reach up to $700 \mathrm{Whr} \cdot \mathrm{kg}^{-1}$ and rechargeable batteries up to $300 \mathrm{Whr} \cdot \mathrm{kg}^{-1}$ [15]. It is also found that, for all operating temperatures in the range $1000-1300 \mathrm{~K}$, the threshold value of $25 \mathrm{ppm}$ for $\mathrm{NO}$ is attained, whereas the residual concentration of ammonia is negligible.

The effect of temperature on the performance of the system results from a trade-off between the heat losses and the chemical/electrochemical kinetics. On one hand, as temperature increases, so do the chemical/electrochemical reaction rates. In other words, increasing the operating temperature allows one to obtain the same conversions while significantly reducing the size of the units. This behavior is illustrated by the blue dotted curve on the right plot of Fig. 9.3, where only the optimal fuel cell volume has been represented since it is much larger than the volumes of the other three units at all temperatures. On the other hand, the heat losses per unit area are substantially increased by operating the system at a higher temperature. In this case study, it is found that an increase in the operating temperature actually decreases the heat losses of an optimized device in the range $1000-1300 \mathrm{~K}$, as illustrated by the red curve on the right plot of Fig. 9.3. In other words, the additional heat losses per unit area incurred by a higher operating temperature are compensated by a reduction in the optimized device size. This rather counter-intuitive result explains why a higher operating temperature is beneficial to the fuel energy density achievable by the system. Note also the very large values of heat- 
losses-to-electrical-power ratio (the device produces $7 \mathrm{~W}$ of heat losses for $1 \mathrm{~W}$ of electrical power at $1000 \mathrm{~K} !$ ), which indicates that a large part of the fuel mass is used to maintain the stack at the prescribed operating temperature.

Regarding operational variables, the optimal value of the air flow rate $F_{\text {in }}^{\mathrm{b}_{\mathrm{I}}}$ in burner I is found to be zero, irrespectively of the stack temperature; in other words, it is more efficient to provide all the oxygen needed for the oxidation reactions in burner I through the fuel cell cathode. It is also found that the optimal flow rates of ammonia, air and butane are relatively unaffected by the operating temperature in comparison to the unit volumes; these values decrease by $22 \%, 35 \%$ and $37 \%$, respectively, between $1000 \mathrm{~K}$ and $1300 \mathrm{~K}$ - which should be compared to the 4 -fold decrease of the device volume. The higher consumption of ammonia and butane fuels at lower operating temperatures is directly linked to the decrease of the fuel energy density.

Effect of the Nominal Power Demand The results presented previously are all relative to an electrical power production of $1 \mathrm{~W}$. It is however legitimate to raise the question how the optimal design and operation of the system scales when a power production as low as $0.1 \mathrm{~W}$ or, conversely, as high as $10 \mathrm{~W}$ is considered. This paragraph provides an overview of that important aspect of the process; compare also Chapter [Selection of Alternatives and Process Design].
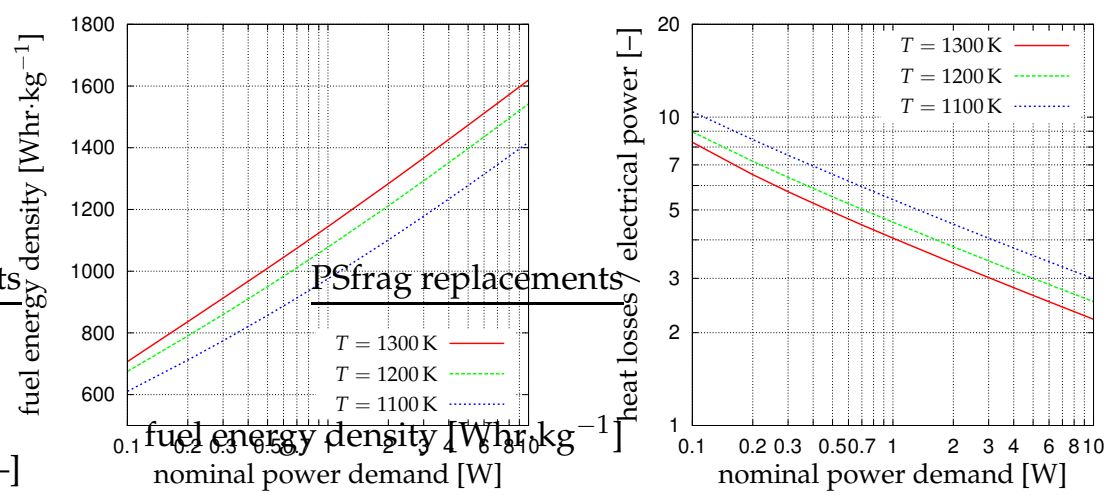

Fig. 9.4: Fuel energy density (left plot) and heat-losses-to-electrical-power ratio (right plot) vs. nominal power demand, for various operating temperatures.

The effect of the nominal power demand $\mathcal{P}^{\text {nom }}$ on the fuel energy density is shown in the left plot of Fig. 9.4, within the range $[0.1 \mathrm{~W}, 10 \mathrm{~W}]$. The three curves in this plot correspond to operating temperatures of $1100 \mathrm{~K}, 1200 \mathrm{~K}$ and $1300 \mathrm{~K}$. One first sees that the nominal power specification has a very large effect on the performance of the system. At $T=1300 \mathrm{~K}$, for instance, the fuel en- 
ergy density varies from $700 \mathrm{Whr} \cdot \mathrm{kg}^{-1}\left(\mathcal{P}^{\text {nom }}=0.1 \mathrm{~W}\right)$ up to $1600 \mathrm{Whr} \cdot \mathrm{kg}^{-1}$ $\left(\mathcal{P}^{\text {nom }}=10 \mathrm{~W}\right)$. It is also seen from this figure that operating the system at $1300 \mathrm{~K}$ always yields the highest energy densities, irrespectively of the nominal power demand.

The relative increase of the heat losses at lower nominal power demands (Fig. 9.4, right plot) is directly related to the size of the device itself. In fact, the optimal values of the unit volumes $V^{\mathrm{r}}, V^{\mathrm{fc}}, V^{\mathrm{b}_{\mathrm{I}}}$, and $V^{\mathrm{b}}$ II scale nearly linearly with $\mathcal{P}^{\text {nom }}$, whereas the heat losses vary proportionally to $\left(V^{\mathrm{r}}+V^{\mathrm{fc}}+V^{\mathrm{b}}+\right.$ $\left.V^{\mathrm{b}_{\text {II }}}\right)^{2 / 3}$. Overall, the the heat-losses-to-electrical-power ratio of the device thus varies proportionally to $\left(V^{\mathrm{r}}+V^{\mathrm{fc}}+V^{\mathrm{b}_{\mathrm{I}}}+V^{\mathrm{b}_{\mathrm{II}}}\right)^{-1 / 3}$.

Regarding operational variables, the flow rates of ammonia (in the reactor) and air (in the fuel cell cathode) are found to be nearly proportional to the power demand. It is also found that the increase in the butane flow rate (vs. the power demand) is slower than that of the ammonia flow rate; this is because relatively more butane is needed at lower power demands to compensate for the additional heat losses.

According to the above considerations, it appears that man-portable power generation systems based on high temperature fuel cells, such as the one shown in Fig. 9.1, will most likely be best suited for applications above $1 \mathrm{~W}$. Below this limit, the relative heat losses indeed become so important that these systems may no longer be competitive with existing batteries. These results corroborate those obtained from system-level analysis (see Chapter [Selection of Alternatives and Process Design]).

Sensitivity to Uncertain Kinetic Rates and Resource Allocation The mathematical expressions used to estimate the kinetic rates in the different unit operations are among the principal sources of uncertainty carried by an intermediate-fidelity model. The following case study considers the influence of uncertain kinetic rates on the optimal design and performance of the fuel cell stack. Here, uncertainty in the kinetic rates is simply represented by varying the pre-exponential factors in the kinetic expressions relative the chemical/electrochemical reactions in the fuel-processing reactor, the anode and cathode compartments of the SOFC, and the two burners. The nominal power demand is set to $\mathcal{P}^{\text {nom }}=10 \mathrm{~W}$ and the operating temperature to $T=1100 \mathrm{~K}$.

Post-optimal sensitivity analysis is used to quantify the effect of this uncertainty on the optimal values of the unit volumes. The results are presented on Fig. 9.5 in the form of histograms. For example, in the histogram labeled ' $I n$ fluence of $\mathrm{NH}_{3}$ decomposition', each bar represents the variation of a given unit's optimal volume in response to a variation in the kinetic rate, $r^{\mathrm{r}}$, of $\mathrm{NH}_{3}$ decomposition (e.g., $\left.\frac{r^{\mathrm{r}} \partial V^{\mathrm{r}}}{V^{\mathrm{r}} \partial r^{\mathrm{r}}}\right)$. Note that the sensitivity coefficients for the unit volumes are typically negative, since an increase in kinetic rate usually allows to reduce 
$18 \mid 9$ Optimal Design and Steady-State Operation
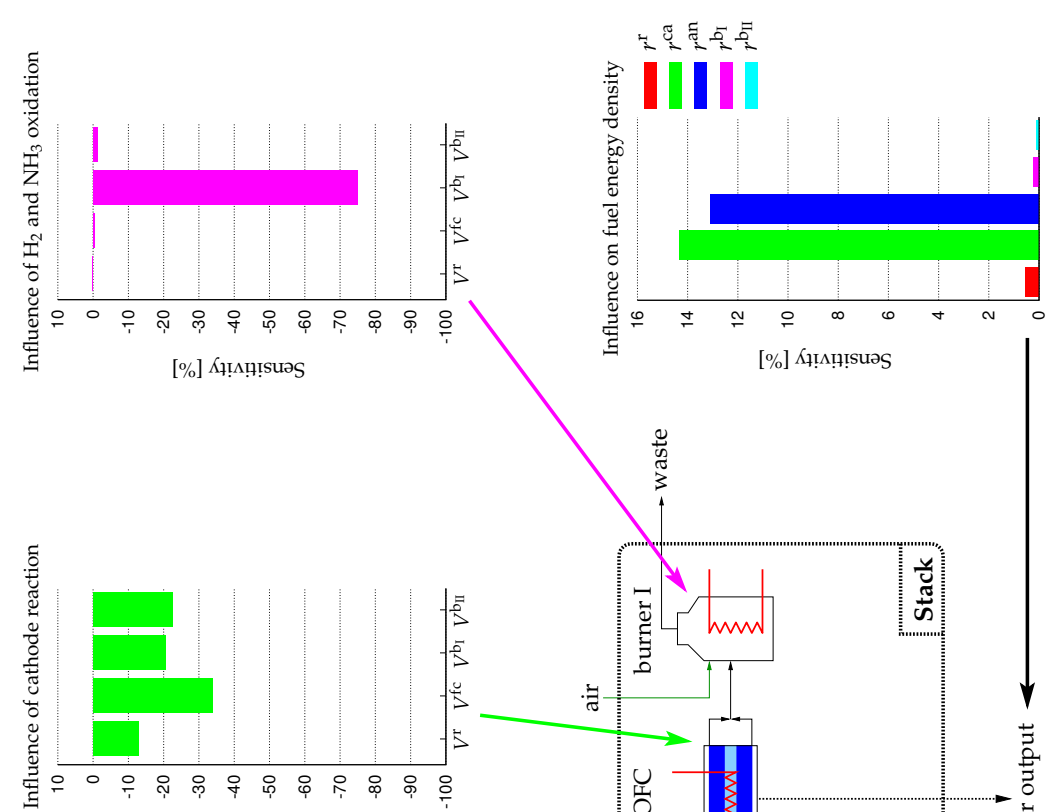

[\%] Kł!

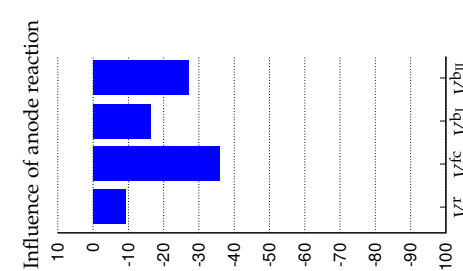

[\%] Kł!n!̣!suas

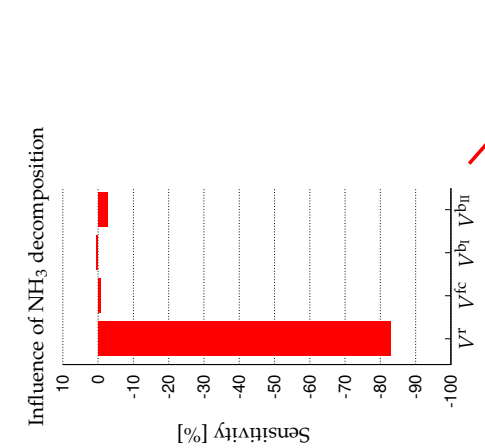

[\%] Kł!

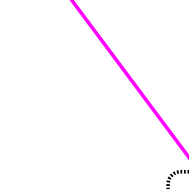

苋
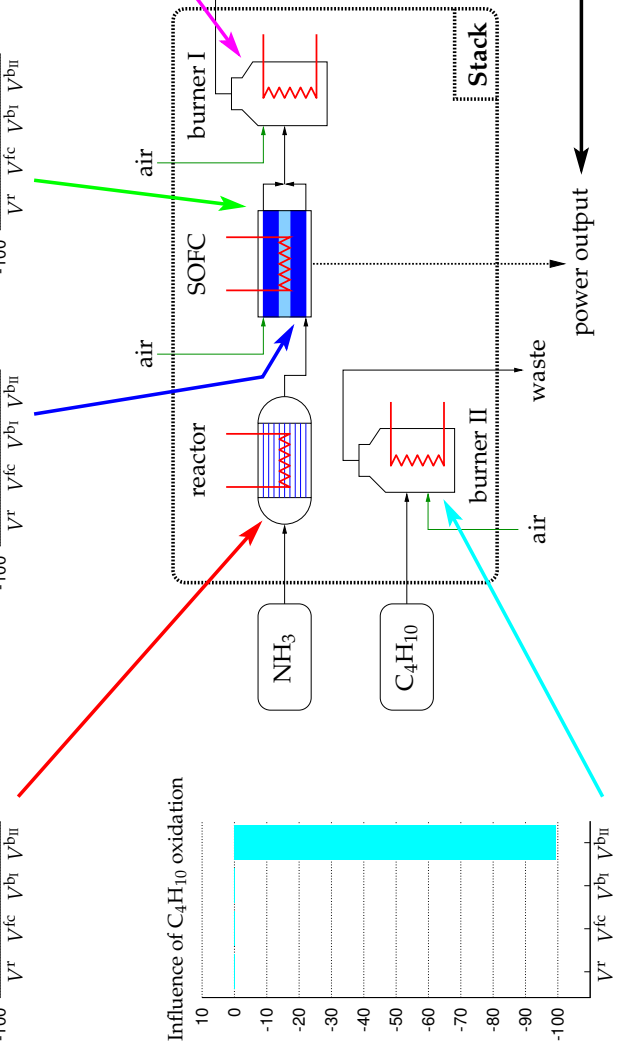

[\%] Kł!

Fig. 9.5: Influence of uncertain reaction rates on the optimal values of the decision variables, for a nominal power demand $\mathcal{P}^{\text {nom }}=10 \mathrm{~W}$ and operating temperature $T=1100 \mathrm{~K}$. 
the volume needed to achieve a given conversion; positive sensitivity coefficients are however possible, such as the influence of $\mathrm{NH}_{3}$ decomposition on $V^{\mathrm{b}_{\mathrm{I}}}$ since a larger production rate of $\mathrm{H}_{2}$ leads to a larger $\mathrm{H}_{2}$ residual concentration at the anode outlet, which in turn justifies a larger burner to generate more heat from the catalytic oxidation of $\mathrm{H}_{2}$. It is seen that a variation in the kinetic rates of $\mathrm{NH}_{3}$ decomposition, $\mathrm{H}_{2}$ and $\mathrm{NH}_{3}$ oxidation, or $\mathrm{C}_{4} \mathrm{H}_{10}$ oxidation, has a very large effect of the optimal volume of the reactor, burner I, or burner II, respectively, but its influence on the volume of other units is very low. On the other hand, a variation in the kinetic rates of electrochemical reaction either at the anode or cathode of the SOFC has a large influence, not only on the optimal volume of the SOFC, but also on the optimal volumes of the reactor, burner I, and burner II. Accordingly, more emphasis should be placed on obtaining accurate chemical kinetics for the micro SOFC than for the other unit operations.

Post-optimal sensitivity analysis is also useful to monitor the influence of the kinetic rates in terms of the system performance. These results are reported in the histogram labeled 'Influence on fuel energy density' on Fig. 9.5. For example, the green bar represents the variation in energy density incurred by a variation in the kinetic rate of electrochemical reaction at the cathode side of the SOFC. The largest sensitivity coefficients, in the order of $13-14 \%$, correspond to the kinetic rates at both the anode and cathode of the SOFC. The other sensitivity coefficients are found to be very small, with values less than $1 \%$. Consequently, if one wants to obtain a reliable estimate of the optimal fuel energy density that can be achieved by the process, special care should clearly be taken for developing accurate kinetic models for either half-cell reactions in the SOFC.

The foregoing sensitivity results also provide useful insight to guide the resource allocation problem for component optimization. Suppose, for example, that we be asked to identify which catalyst should be improved in priority to enhance the system performance. Note first that the main reason for improved performance is that faster kinetics result in smaller residence time requirements and, therefore, lower heat losses, because of the decreased device size. And because sensitivity analysis has shown that the process performance is mostly sensitive to the fuel cell kinetics, the priority should obviously be given to improving both electrodes simultaneously.

\section{4}

\section{Optimal Design and Steady-State Operation for Variable Power Demand}

Most portable electronic devices are not operated at a constant power demand. Not only the magnitudes, but also the durations of operation at dif- 
ferent levels vary. For example, a cellular phone on stand-by mode expends only a small fraction of the power needed during a conversation; the power demand of a portable computer can range between $5 \mathrm{~W}$ and $30 \mathrm{~W}$ depending on usage; the electronic equipment of the dismounted soldier is expected to require $20 \mathrm{~W}$ of power with a peak demand of $50 \mathrm{~W}$.

In this section, the optimal design and steady-state operation of devices having a variable power demand is addressed. For simplicity, it is assumed that the power demand can be approximated well through a small number of discrete power demands and associated demand frequencies; such an approximation can be made in many practical applications, e.g., in the list of applications reported previously.

In a variable power demand problem, the demand, in addition to stringent requirements on the operation of the device stemming from reliability and safety considerations, need to be satisfied for all foreseeable power demand scenarios. In particular, a design based on a single nominal power demand (e.g., as determined previously in Section 9.3) cannot guarantee that such requirements are met for multiple power levels. A conceptually simple way to account for this power variation is to consider the time profile and formulate a dynamic optimization problem based on a transient model. However, this approach is computationally expensive. A more elegant and computationally more tractable approach based on two-stage stochastic programming is used subsequently [27]. This formulation has the advantage that only the average time spent at each power demand is needed, in contrast to a detailed transient power profile. A limitation, however, is that the penalty incurred by switching from one power output level to another must be negligible as well as the duration of transient operation compared to steady-state operation. It is well known that fuel cell based power generation systems are not responsive enough to meet rapid demand changes and, consequently, batteries and/or super-capacitors need to be deployed to work in tandem to meet this deficiency (see Chapter [Design of Hybrid Electrochemical Devices]). In such hybrid electrochemical systems, microfabricated fuel cells are therefore intended to operate mostly at steady state, which justifies the foregoing assumptions.

In a two-stage programming formulation, a distinction is made between the design decision variables and the operational decision variables. Essentially, the design decision variables (also called first-stage variables) represent properties of the system that cannot be altered after fabrication of the device (e.g., volume of reactor or fuel cell), while the operational decision variables (also called second-stage variables) represent properties of the system that can be adapted to the current power demand (e.g., operating temperature, cell voltage or flow rates). 
- For a given power demand scenario and a specified process design, optimal values for the operational variables can be determined by solving an optimization problem of the form:

"Determine the operational decisions that maximize the system performance subject to safety and operational constraints."

Note that the latter problem is similar to those solved in Section 9.3 for a nominal power demand, as if the design variables were fixed.

- Optimal values for the design variables, on the other hand, can be determined from the solution of a higher-level optimization problem of the form:

"Determine the design decisions that maximize the system performance over the set of all possible power demand scenarios, and such that feasible operational decisions can be found for each power demand scenario."

Typically, the performance to be maximized in this problem consists of two contributions: (i) a design cost dependent only on the design variables; (ii) an operating cost obtained by averaging the operating costs for all foreseeable power demand scenarios. Note that, for each scenario, a set of optimal operational variables is to be determined. In particular, all these operational strategies must satisfy the safety and operational constraints (as described earlier in Section 9.3). Therefore, solving the design and steady-state operation problem in the context of a variable power demand is much more involved than in the nominal case.

Once an optimal design and operation strategy has been identified, parametric studies based either on post-optimal sensitivity analysis (local analysis) or parametric programming (global analysis) can be applied to assess the level of confidence that can be placed in that strategy.

Optimal design and steady-state operation for variable power demand is illustrated by a case study below.

\section{Case Study (Continued)}

The man-portable power generation process shown in Fig. 9.1 is considered, along with the intermediate-fidelity model described earlier in Section 9.2. It is supposed here that a finite number of possible power demands, $\mathcal{P}_{i}^{\text {nom }}$, $i=1, \ldots, N_{d}$, can occur during the time frame of interest, with corresponding frequencies of occurrence, $\omega_{i}^{\text {nom }}$. The two-stage programming formulation for optimal design and steady-state operation is to determine the values of the design variables $V^{\mathrm{r}}, V^{\mathrm{fc}}, V^{\mathrm{b}}$ and, for each possible power demand $\mathcal{P}_{i}^{\text {nom }}$, $i=1, \ldots, N_{d}$, the values of the operational variables $T_{i}, U_{i}, F_{\mathrm{in}, i^{\prime}}^{\mathrm{r}}, F_{\mathrm{in}, i^{i}}^{\mathrm{ca}}, F_{\mathrm{in}, i^{i}}^{\mathrm{b}_{\mathrm{I}}}, F_{\mathrm{in}, i}^{\mathrm{b}_{\text {II }}}$ 
that

maximize : mission fuel energy density

subject to : nominal power demand autothermal operation for each scenario maximum $\mathrm{NH}_{3}$ and $\mathrm{NO}$ emissions $\} i=1, \ldots, N_{d}$.

A number of remarks are in order:

- The mathematical expressions for the constraints are the same as those given by Equations (9.5) to (9.8).

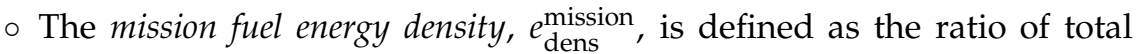
energy produced to the amount of fuel required, normalized by the mission duration. Letting $\mathcal{M}_{i}$ denote the mass flow rate of fuels in the device corresponding to the power demand $\mathcal{P}_{i}^{\text {nom }}$, one has:

$$
e_{\text {dens }}^{\text {mission }}:=\sum_{i=1}^{N_{d}} \omega_{i}^{\text {nom }} \mathcal{P}_{i}^{\text {nom }} / \sum_{i=1}^{N_{d}} \omega_{i}^{\text {nom }} \mathcal{M}_{i}
$$

This objective is appropriate for applications where the mass of the fuel to be carried dominates over the total mass of the device, or where refueling is infrequent due to logistic restrictions. The numerator of (9.9) being constant for given frequencies and values of the power demand, maximizing $e_{\text {dens }}^{\text {mission }}$ is equivalent to minimizing the sum of mass flow rates weighted by the frequencies.

In this case study, the design of a system to power the electronic equipment of the dismounted soldier is considered. The power demand can take two possible values: $20 \mathrm{~W}$ and $50 \mathrm{~W}$, with the former occurring $90 \%$ of the time [24]. Moreover, the maximum operating temperature for the system is set to $1100 \mathrm{~K}$. The performance of a design for nominal power demand is first considered, then the results obtained with the two-stage programming approach are presented and compared to other design approaches.

Performance of Nominal Power Demand Design In this paragraph, the system is designed based on nominal power demand considerations: first, a nominal power demand is selected and the system is designed for that particular demand disregarding operation at any other demand; then, this design is fixed and optimal operational decisions are determined for the various power demand scenarios. The following approaches are investigated:

- Design for Average Power Demand The system is designed for the power demand $\mathcal{P}^{\text {ave }}:=\frac{1}{N_{d}} \sum_{i=1}^{N_{d}} \mathcal{P}_{i}^{\text {nom }}=35 \mathrm{~W}$. 
- Design for Maximum Power Demand The system is designed for the power demand $\mathcal{P}^{\max }=50 \mathrm{~W}$.

For performance assessment, the resulting designs are compared to the ideal design, which represents a design where the component volumes can be adjusted optimally for each possible power demand. Clearly, this ideal design has the best performance, but it is not realizable.

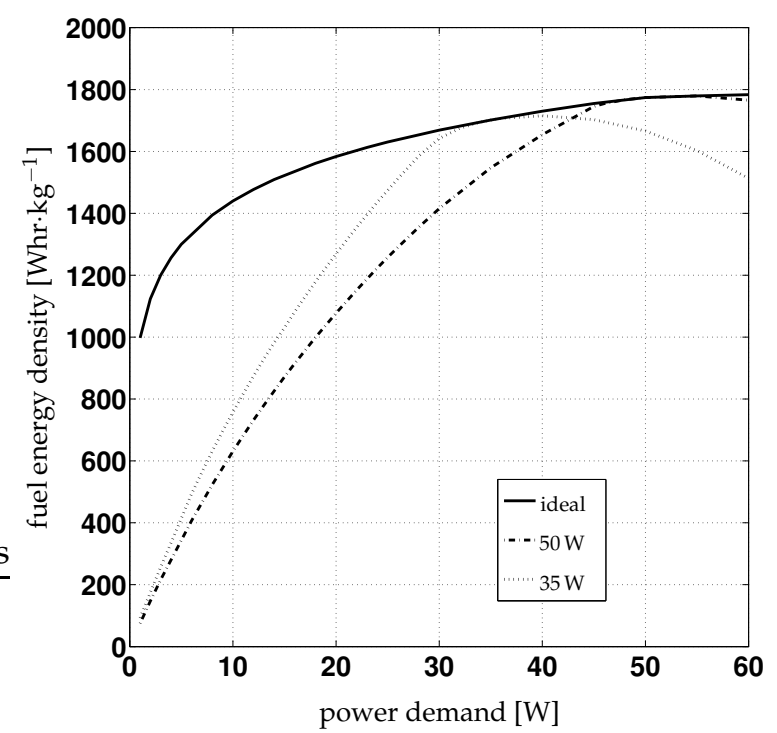

Fig. 9.6: Performance of fuel cell stack designs for average power demand ( $35 \mathrm{~W})$ and maximum power demand $(50 \mathrm{~W})$, for the dismounted soldier application.

The results are shown in Fig. 9.6. Note that the performances of the designs for average and maximum power vary significantly over the power demand range of interest. As expected, the curves for these designs intersect the ideal design curve at $35 \mathrm{~W}$ and $50 \mathrm{~W}$ demands, respectively. In either case, however, performance degrades to unacceptable levels when the actual demand and the design demand differ significantly.

In case the actual demand is less than the design demand, the total volume of the device is larger than the volume of the optimal design for that demand. More heat losses occur than is ideal during operation to meet the actual demand and a larger amount of butane than ideally required is thus needed to keep the stack at a constant operating temperature. Accordingly, a decrease in the performance is observed.

In case the actual demand is higher than the design demand, the volumes are smaller than the optimal volumes for that demand. Smaller volumes lead 
to lower conversions in the reactor and fuel cell. In turn, lower conversions are compensated by a higher flow rate of ammonia to provide the necessary $\mathrm{H}_{2}$ to meet the power demand. The mole fractions of $\mathrm{NH}_{3}$ and $\mathrm{NO}$ in the effluent increase as higher flow rates and smaller volumes result in lower residence times in the units. Larger air flow rates are then required to dilute the mole fraction of $\mathrm{NH}_{3}$ and $\mathrm{NO}$ to acceptable and safe levels. Even though heat losses decrease, more $\mathrm{C}_{4} \mathrm{H}_{10}$ is therefore required in order to heat up this additional air. Overall, the flow rates of ammonia and butane increase, and a decrease in the performance is obtained. Even more problematic, as the efficiency of the butane burner decreases with increased $\mathrm{C}_{4} \mathrm{H}_{10}$ flow rate (due to shorter residence times), a demand is reached eventually for which the emission constraints on $\mathrm{NH}_{3}$ and $\mathrm{NO}$ can no longer be met by increasing the air flow rates while operating autothermally. For this reason, it not feasible to produce, e.g., $50 \mathrm{~W}$ of electrical power with a $20 \mathrm{~W}$ nominal power demand design.

Performance of Two-Stage Programming Design The significant degradation in performance of a nominal power demand design when the actual demand is far from the nominal one motivates the application of the two-stage programming approach to determine design and operational decision variables.

The comparison of the two-stage programming approach with the foregoing nominal power demand designs, in terms of the mission fuel energy density (9.9), is presented in Fig. 9.7. Also shown on this figure is the performance of the so-called conservative design for mean power demand, which aims to optimize the mean power demand during the mission, $\mathcal{P}^{\text {mean }}:=$ $\sum_{i=1}^{N_{d}} \omega_{i}^{\text {nom }} \mathcal{P}_{i}^{\text {nom }}=23 \mathrm{~W}$, while guaranteeing that the selected design is feasible at all the power demand levels.

The difference between the ideal design and the two-stage design is only $100 \mathrm{Whr} \cdot \mathrm{kg}^{-1}$. In particular, the two-stage design allows to recover a significant portion of the best theoretical possible performance. It is found that this design results in $25 \%$ lower fuel expenditure than the maximum power demand design, and 13\% lower fuel expenditure than either the conservative mean power demand or the average power demand design. More precisely, it appears that the reduction in fuel expenditure due to the two-stage design is significantly better than at lower power demands than it is at higher power demands. This is a direct result of the fuel energy density varying more rapidly with demand at lower power demands.

\section{Acknowledgment}

This work was supported by the DoD Multidisciplinary University Research Initiative (MURI) program administered by the Army Research Office under 


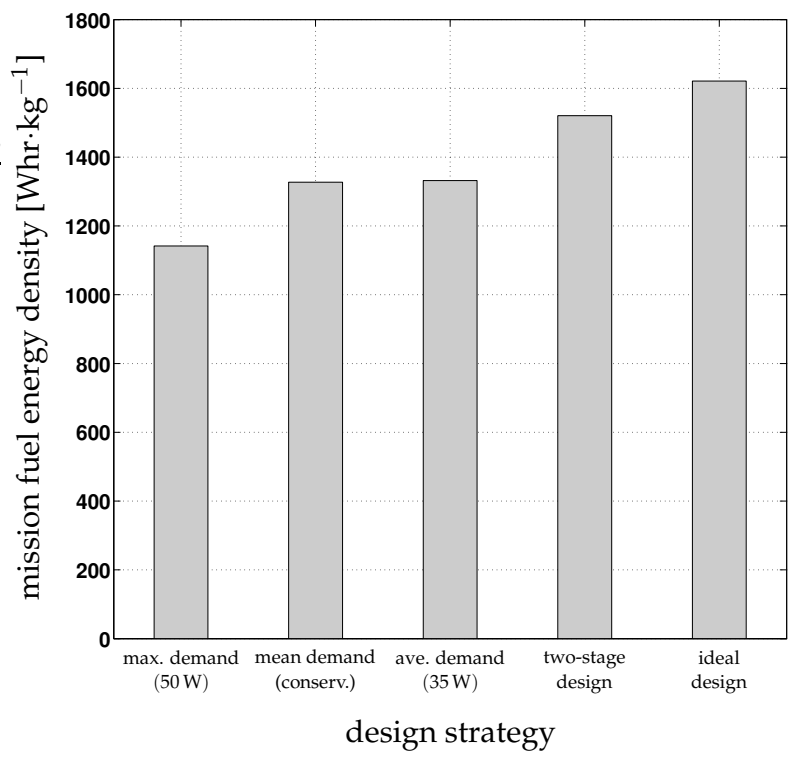

Fig. 9.7: Comparison of various design strategies for the dismounted soldier application.

Grant DAAD19-01-1-0566. The author would like to acknowledge P. I. Barton, A. Mitsos, and M. Yunt for their contributions which led to this chapter.

\section{Bibliography}

1 Achenbach, E. (1994). Three-dimensional and time-dependent simulation of a planar solid oxide fuel cell. J. Power Sources, 49:333-348.

2 Arana, L. R., Baertsch, C. D., Franz, A. J., Schmidt, M. A., and Jensen, K. F. (2003). A microfabricated suspended-tube chemical reactor for thermally efficient fuel processing. IEEE J. MEMS, 12(5):600-612.

3 Bank, B., Guddat, J., Klatte, D., Kummer, B., and Tammer, K. (1983). Non-Linear Parametric Optimization. Birkhäuser Verlag, Basel.

4 Barton, P. I., Mitsos, A., and Chachuat, B. (2005). Optimal start-up of micro power generation processes. In Puigjaner, L. and Espuña, A., editors, Computer Aided Chemical Engineering, volume 20B, pages 1093-1098, The Netherlands. Elsevier
5 Bessette, II, N. F., Wepfer, W. J., and Winnick, J. (1995). A mathematical model of a solid oxide fuel cell. J. Electrochem. Soc., 142(11):3792-3800.

6 Biegler, L. T. and Grossmann, I. E. (2004). Retrospective on optimization. Comput. Chem. Eng., 28(8):1169-1192.

7 Buskens, C. and Maurer, H. (2000). SQPmethods for solving optimal control problems with control and state constraints: adjoint variables, sensitivity analysis and real-time control. J. Comput. Appl. Math., 120(1-2):85-108.

8 Chachuat, B., Mitsos, A., and Barton, P. I. (2005a). Optimal design and steadystate operation of micro power generation employing fuel cells. Chem. Eng. Sci., 60(16):4535-4556. 
9 Chachuat, B., Mitsos, A., and Barton, P. I. (2005b). Optimal start-up of micro power generation processes employing fuel cells. In AIChE Annual Meeting, 30 Oct-4 Nov 2005, Cincinnati, OH.

10 Deshmukh, S. R., Mhadeshwar, A. B., and Vlachos, D. G. (2004). Microreactor modeling for hydrogen production from ammonia decomposition on ruthenium Ind. Eng. Chem. Res., 43(12):2986-2999.

11 Deshmukh, S. R. and Vlachos, D. G. (2005). Effect of flow configuration on the operation of coupled combustor/reformer microdevices for hydrogen production. Chem. Eng. Sci., 60:5718-5728.

12 Fiacco, A. V. (1983). Introduction to Sensitivity and Stability Analysis in Nonlinear Programming, volume 165 of Mathematics in Science and Engineering. Academic Press, New York.

13 Fogler, H. S. (1998). Elements of Chemical Reaction Engineering. Prentice-Hall, New Jersey, 3rd edition.

14 Hsing, I. M., Srinivasan, R., Harold, M. P., Jensen, K. F., and Schmidt, M. A. (2000). Simulation of micromachined chemical reactors for heterogeneous partial oxidation reactions. Chem. Eng. Sci., 55:3-13.

15 Linden, D. (2001). Handbook of Batteries. McGraw Hill.

16 Mitsos, A. (2006). Man-Portable Power Generation Devices: Product Design and Supporting Algorithms. PhD thesis, Massachusetts Institute of Technology, Cambridge, MA.

17 Mitsos, A., Chachuat, B., and Barton, P. I. (2007a). Methodology for the design of man-portable power generation devices. Ind. Eng. Chem. Res., 46(22):7164-7176.

18 Mitsos, A., Chachuat, B., and Barton, P. I. (2007b). What is the design objective for portable power generation: Efficiency or energy density? J. Power Sources, 164(2):678-687.

19 Mitsos, A., Hencke, M. M., and Barton, P. I. (2005). Product engineering for manportable power generation based on fuel cells. AIChE J., 51(8):2199-2219.

20 Mitsos, A., Palou-Rivera, I., and Barton, P. I. (2004). Alternatives for micropower generation processes. Ind. Eng. Chem. Res., 43(1):74-84

21 Norton, D. G. and Vlachos, D. G. (2004). A CFD study of propane/air microflame stability. Combust. Flame, 138:97-107.

22 Pignet, T. and Schmidt, L. D. (1975). Kinetics of $\mathrm{NH}_{3}$ oxidation on $\mathrm{Pt}, \mathrm{Rh}$ and $\mathrm{Pd}$. J. Catal., 40:212-225.

23 Poshusta, J. C., Kulprathipanja, A., Martin, J. L., and Martin, C. M. (2006). Design and integration of portable SOFC generators. In AIChE Annual Meeting, 12-17 Nov 2006, San Francisco, CA.

24 Soldier Power/Energy Systems Committee (2004). Meeting the Energy Needs of Future Warriors. The National Academies Press, Washington DC.

25 Srikar, V. T., Turner, K. T., Ie, T. Y. A., and Spearing, S. M. (2004). Structural design considerations for micromachined solid-oxide fuel cells. I. Power Sources, 125(1):62-69.

26 Westbrook, C. K. and Dryer, F. L. (1984). Chemical kinetic modeling of hydrocarbon combustion. Proc. Energy Combust. Sci., 10:1-57.

27 Yunt, M., Chachuat, B., Mitsos, A., and Barton, P. I. (2008). Designing manportable power generation systems for varying power demand. AIChE J., 54(5):1254-1269. 